\title{
Application of infrared thermography in investigation of hydrostatic extrusion
}

\author{
T. S. Wisniewski ${ }^{*}$ W. Pachla**, D. Kukla*** , A. Mazur** \\ and K. J. Kurzydłowski ${ }^{* * *}$ \\ *Institute of Heat Engineering, Warsaw University of Technology, Warszawa, Poland \\ **High Pressure Research Center, Polish Academy of Sciences UNIPRESS, \\ Warszawa, Poland \\ ${ }^{* * *}$ Materials Science and Engineering Faculty, Warsaw University of Technology, \\ Warszawa, Poland
}

\begin{abstract}
Hydrostatic extrusion is a technique based on severe plastic deformation. Characteristic features of this process are very large deformation, high strains, strain rates and considerable thermal effect. Temperature is one of the most important factors which controls the structure and mechanical properties of material during this process. Due to very high extrusion pressure (of the order of magnitude $1 \mathrm{GPa}$ ) and high speed of extruded material only the 'contactless' methods of the product temperature measurement during the process are possible. Results of measurements with use of ThermaCAM SC 2000 at recording frequency $50 \mathrm{~Hz}$ are presented in the paper for hydrostatic extrusion of aluminium wires with OD between 2-3 $\mathrm{mm}$.
\end{abstract}

\section{Introduction}

In recent years several techniques based on severe plastic deformation (SPD), leading to generation of nanostructures in metals and alloys, have been developed. These are cyclic extrusion compression (CEC), hydrostatic extrusion $(\mathrm{HE})$, equal channel angular pressing (ECAP) or high pressure torsion (HPT). They all involve thermal effects associated with mechanical work done on the deformed material. This thermal effect is especially pronounced in the case of hydrostatic extrusion (see Figure 1) since high strains are here combined with high strain rates. The billet material surrounded by the high pressure transmitting medium is extruded through the die after the certain pressure level, generated by the piston penetrating the working chamber is attained. Since friction losses are limited to a minimum (no friction at billet/chamber interface) very large deformation per pass (true strains as high as 2 and more), high strain rates $\left(\sim 10^{2}-10^{4} \mathrm{~s}^{-1}\right)$ and a very homogeneous deformation (low angle dies) are achieved during hydrostatic extrusion. High strains and strain rates are always accompanied by significant thermal effect leading to increase of the extruded product temperature.

Temperature is one of the dominating factors which controls the structure and mechanical properties of metals and alloys during their plastic deformation. Thermal effect generated during hydrostatic extrusion may lead to dynamic recrystallization within the extruded product, and results in structure characteristic for hot deformation. In some cases, the extruded product can even be fully recrystallized, which may eliminate the need of post-annealing. Under some circumstances, combination of large strains with thermal effect allows to obtain materials with ultrafine structures or nanomaterials [1,2]. Therefore, it is important to monitor 'on 
line' and control the temperature rise within the deformed product. Ability to predict the temperature increase during plastic deformation of metals and alloys is crucial for control and design of the structures and properties in final products. The magnitude of the thermal effect depends on the extrusion pressure and the material properties

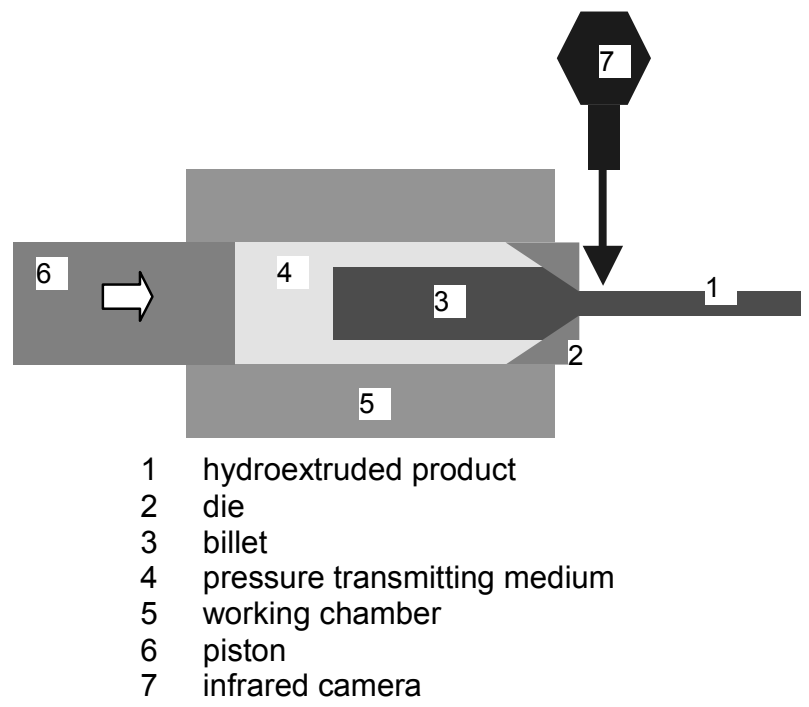

Fig. 1. Principle of the hydrostatic extrusion process and the infrared thermography measurements

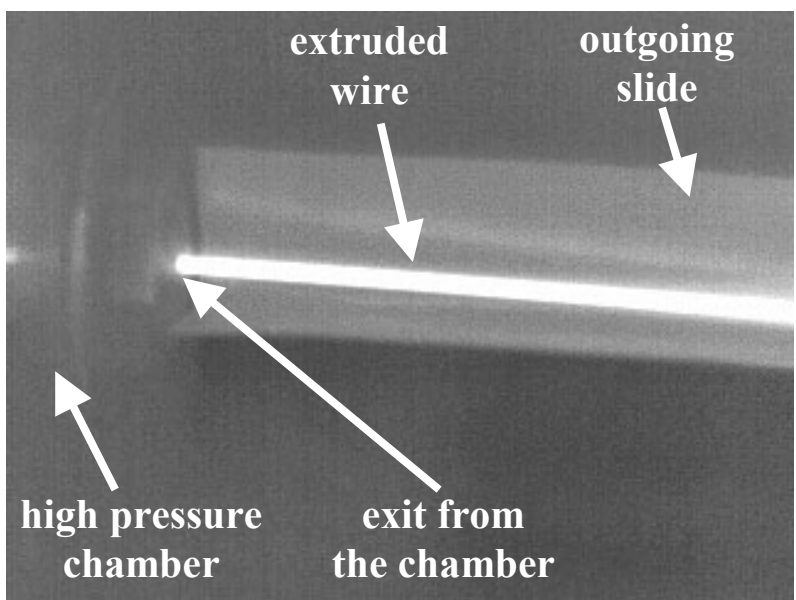

Fig. 2. An example of the IR image of the hydrostatically extruded aluminium wire of $2.2 \mathrm{~mm}$ in $\mathrm{OD}$ 
and can increase the product temperature by hundreds degree centigrages (as in case of aluminium and copper $[3,4]$ ). This increase is caused by mechanical work done on the material, adiabatic compression within the workpiece and friction at the die/product interface. Since the linear speed of extruded products can achieve $10 \mathrm{~m} / \mathrm{s}$ it is possible to measure the temperature rise of the product during the process at the die exit only by the 'contactless' means, Figure 1. Infrared thermography gives chance to measure the fast changes of temperature with high accuracy during such a dynamic process as hydrostatic extrusion. This method can be applied due to the fact, that the extruded product after leaving the extrusion die is directly visible for the infrared camera. Since the hydrostatic extrusion process involves high pressures treatment of the order of magnitude $1 \mathrm{GPa}$ and more, due to the safety reasons, the IR thermography has the additional advantage over any other 'contact' method.

\section{Experimental}

The technical purity aluminium $99.9 \%$ was used in all hydrostatic extrusion experiments. The applied hydrostatic extrusion press enables for extrusion of billets in the range of starting $\mathrm{OD}$ up to $28 \mathrm{~mm}$ and approximately $150 \mathrm{~cm}^{3}$ in volume. The available extrusion pressure range is up to $1.7 \mathrm{GPa}$ at room temperature. The controlled hydraulic supply system allows adjusting the linear speed of the extruded products (wires, tubes, complex shapes of max. OD=10mm) in the range of true strains exceeding 9 (i.e.: reduction ratio of cross sections above 100) in one pass and strain rates approaching $10^{4} \mathrm{~s}^{-1}$.

During IR measurements the outgoing wires have moved with high speed over the guiding slide, so the 'on-line' temperature monitoring along the full length of the extruded product was made. In present study the reduction ratios have ranged between 50 and 150, the measured wire diameters have varied between 2 and $3 \mathrm{~mm}$ in OD and the lengths have reached up to 30 meters with process duration up to 20 s.

The infrared measurements were performed with use of ThermaCAM SC 2000 camera together with the high speed recording interface and ThermaCAM Researcher 2001 software. The images of extruded products were recorded with frequency of $50 \mathrm{~Hz}$. An example of the IR image obtained during the process is shown in Figure 2. The IR measurements were performed during the process of extrusion and following 20-30 s. Immediately after stopping the extrusion process (the wire forward move) measurement of product temperature with use of sheathed thermocouple was made. The thermocouple was clamped to the extruded wire surface along $20 \mathrm{~mm}$ next to the segment measured by IR camera. This contact measurement of temperature was performed simultaneously with IR recording and allowed for estimation of the wire surface emissivity.

\section{Results and discussion}

Typical flow-charts of the extrusion pressure and the temperature rise during hydrostatic extrusion of commercial pure aluminium wires are shown in Figures 3 and 4. The main differences between both cases are in the extrusion pressure and linear extrusion speed. These parameters are $800 \mathrm{MPa}$ vs. $620 \mathrm{MPa}$ and $1.3 \mathrm{~m} / \mathrm{s}$ vs. $0.66 \mathrm{~m} / \mathrm{s}$, respectively.

Independent on these differences, the characteristic peak in temperature has been observed at the start-up, which can be attributed to boundary friction at the beginning of the process. It is followed by the sudden drop in temperature when the extruded material approaches the steady flow condition and low friction reflecting the 


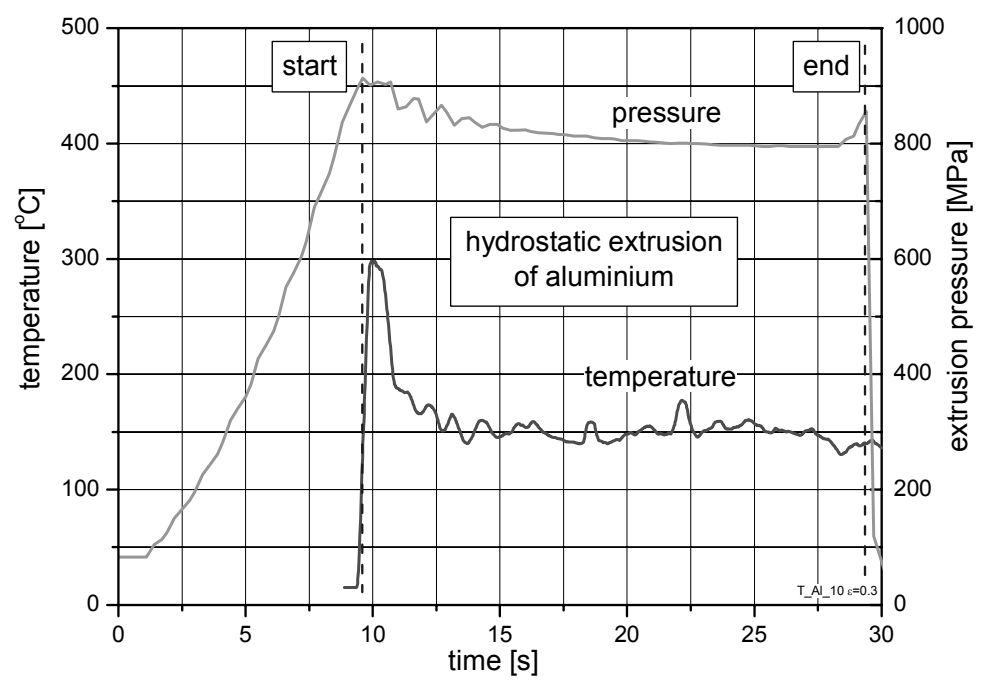

Fig. 3. Extrusion pressure and temperature rise during hydrostatic extrusion of aluminium wire $O D=2.2 \mathrm{~mm}$ with reduction $R=98$; linear extrusion speed $1.3 \mathrm{~m} / \mathrm{s}$

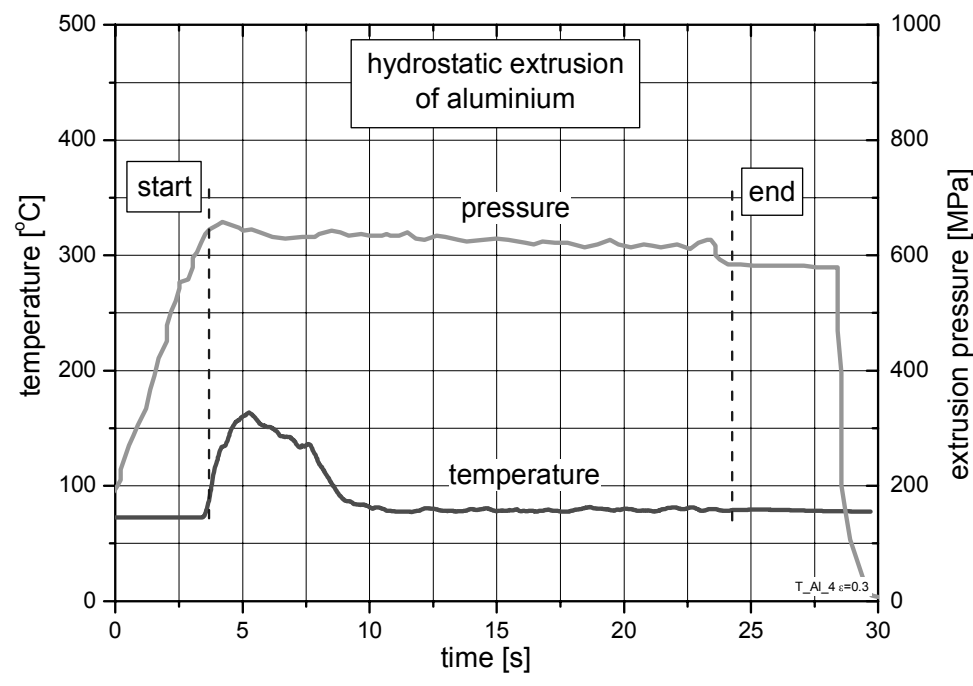

Fig. 4. Extrusion pressure and temperature rise during hydrostatic extrusion of aluminium wire $O D=2.88 \mathrm{~mm}$ with reduction $R=88$; linear extrusion speed $0.66 \mathrm{~m} / \mathrm{s}$ 
hydrodynamic lubrication. For higher extrusion speed the 'start-up' temperature peak is higher.

In hydrostatic extrusion the billet nose is conically tapered to seal the die before the process begins. Therefore, the extrusion conditions (strain, strain rate and friction) at the start-up differ substantially from the steady-state ones. This causes, that the initial portion of the product (1 -2 meters) is covered with different lubricant layer (thicker) then the rest of the product. This portion has much higher emissivity than the rest of the extruded wire. Therefore, at least two different emissivities must be taken into consideration for these two parts of the product. Figures 3 and 4 have been plot for $\varepsilon=0.3$ characteristic for the later (steady-state) portion of the wire, and the observed peaks at the start-up of the process will not be further discussed.

The second apparent observation is, that the temperature rise is higher for higher extrusion pressure. Practically, it was observed for all specimens. This can be attributed to the 'adiabatic' heating of the deformed material resulting from the mechanical work done during hydrostatic extrusion [4].

The third observation is, that the higher extrusion speed resulted in evident temperature oscillation, Figure 3, while for lower speed the temperature remains almost constant during the duration of the process, Figure 4. Please note, that in the first case the linear extrusion speed was doubled. The observed temperature oscillation may be attributed to the local changes in emissivity resulting from noneven distribution of the lubricant at the measured product surface at higher speeds. The clearily visible darker and lighter spots have been observed at the surfaces of some wires, especially those deformed at higher speeds. These temperature oscillations lasted during full extrusion length, despite the fact, that the extrusion pressure has monotonously decreased.

Obviously, the emissivity of aluminium wire surface depends on the billet surface preparation technique, the surface quality itself and on the applied lubricant. For both types of lubricants applied the emissivities of hydrostatically extruded commercial purity $99.9 \%$ aluminium, in 'steady-state' process condiction, were evaluated for different specimens between 0.15 and 0.3 .

The final observation is, that the magnitude of temperature rise during hydrostatic extrusion of aluminium is quite significant in comparison to recrystallization temperature $\left(350^{\circ} \mathrm{C}-450^{\circ} \mathrm{C}\right)$, and can results in significant transformation within the microstructure of extruded wires. Starting with the cold billet (at room temperature) has led to the product temperature of $\sim 80^{\circ} \mathrm{C}$ for lower extrusion ratio $(R=88)$ and $\sim 150^{\circ} \mathrm{C}$ for higher ratio $(R=98)$. The later effect has been additionally reinforced by the doubled extrusion speed in comparison to the first case.

\section{Conclusions}

The infrared thermogarphy is a sensitive method which can be successfully utilized for measurements of the temperature rise during the ultra-high speed metalforming processes, such as the hydrostatic extrusion. At the beginning of the process due to the variable process conditions (acceleration, reduction ratio, lubrication) the surface emissivity drastically differs from the emissivity values for the steady-state run. The later characterizes in substantial temperature increase, higher for the higher extrusion pressure. Temperature rise in aluminium can be as high as $150^{\circ} \mathrm{C}$ for reduction ratio $\sim 100$. For higher extrusion speeds local changes in emissivity at the wire surface are observed, what is attributed to non-even distribution of thinner lubricant film. 


\section{REFERENCES}

[1] Lewandowska, M., Garbacz, H., Pachla W., Mazur A., Kurzydłowski, K. J. Hydrostatic Extrusion and Nanostructure Formation in an Aluminium Alloy, Solid State Phenomena, in press (2004)

[2] Jeong, H. G., Yoon, D. J., Kim, E. Z., Park, H. J. J. Mater. Process. Technol. vol. 438, 130-131 (2002)

[3] Styczyński, L., Pachla W., Wojciechowski, S. Thermal Softening Processes in Polycrystalline Aluminum During Hydrostatic Extrusion, Metal Science, vol. 16, 525-528 (1982)

[4] Pachla, W., Styczynski, L., Porowski, S., Wojciechowski, S. Recovery and Recrystallization of Polycrystalline Copper During Hydrostatic Extrusion, Metal Science, vol. 16, 519-524 (1982)

\section{Acknowledgment}

This work was supported by the Ministry of Scientific Research and Information Technology under grant No 3T08B 03526 and grant No 4T08A 04525. 